\title{
O USO DE CORTISOL SALIVAR COMO MARCADOR BIOLÓGICO PARA O STRESS EM PESQUISAS DE COMPORTAMENTO DO CONSUMIDOR
}

\begin{abstract}
Objetivo: Este estudo buscou validar o protocolo de estímulo de stress psicológico em grupos - G-TSST -, a medida de cortisol salivar para estudos de stress em comportamento do consumidor e confrontar a medida biológica com outras subjetivas e de percepção.

Método: Através de design experimental 2x1 com manipulação between subjects, foram avaliadas as respostas fisiológicas e de percepção para stress, em 50 sujeitos divididos entre grupo controle e experimental, submetidos ao G-TSST.

Originalidade/Relevância: A manipulação laboratorial de stress em um estudo de marketing, a utilização da medida salivar de cortisol - hormônio secretado no organismo humano em situações de stress - e seu confronto com medidas clássicas de percepção e auto relato são inéditas no campo.

Resultados: Os resultados indicam que o protocolo foi eficiente em gerar o estado de stress agudo nos participantes, a medida de cortisol salivar é válida e confiável para o proposto e as medidas subjetivas e biológica não são correlacionadas e medem objetos distintos, sendo, portanto, não substituíveis.

Contribuições teóricas/metodológicas: A importância da investigação sobre os impactos do stress no comportamento do consumidor é ressaltada neste estudo com a validação de um protocolo de estímulo e da inclusão de medidas fisiológicas, em especial salivares, como opção viável aos pesquisadores de marketing. Além disso é discutida a discrepância entre os resultados encontrados na medida fisiológica de stress e naquelas de percepção/auto relato.
\end{abstract}

Palavras-chave: Comportamento do Consumidor. Stress. Cortisol Salivar.

\section{THE USE OF SALIVARY CORTISOL AS A BIOLOGICAL MARKER FOR STRESS IN CONSUMER BEHAVIOR RESEARCH}

Objective: This study aimed to validate a protocol for psychological stress in groups - G-TSST -, the measurement of salivary cortisol for stress studies on consumer behavior and to compare the biological measure with subjective and perceptual ones.

Method: Through 2x1 experimental design with between-subjects manipulation, physiological and perceptual responses to stress were measured in 50 subjects (control and experimental group), submitted to G-TSST.

Originality / Relevance: Both the manipulation of stress in a marketing study, the use of a salivary measure of cortisol - a hormone secreted in the human body in acute stress situations - and its confrontation with classic paperand-pencil perception measures are new in the field.

Results: The results indicate that G-TSST was efficient in generating acute stress in the participants, the salivary cortisol measurement is valid and reliable for the objective and that the subjective and biological measurements are not correlated and not replaceable.

Theoretical/methodological contributions: The importance of studies about stress on consumer behavior is highlighted in this research with the validation of a stress induction protocol and the inclusion of physiological measures, especially a salivary one, as a viable option for marketing researchers. In addition, we discuss the discrepancy between results for physiological and perceived stress measures.

Keywords: Consumer Behavior. Stress. Salivary Cortisol.

Cristina Maria de Aguiar Pastore ${ }^{1}$ Eliane Cristine Francisco-Maffezzolli ${ }^{2}$

\footnotetext{
${ }^{1}$ Doutoranda em Administração de Empresas pela Pontifícia Universidade Católica do Paraná - PUC/PR. Professora da Pontifícia Universidade Católica do Paraná - PUC/PR. Paraná, Brasil. E-mail: cristina.pastore@pucpr.br

${ }^{2}$ Doutora em Administração de Empresas pela Universidade Federal do Paraná - UFPR. Professora do Programa de Pós-graduação em Administração da Pontifícia Universidade Católica do Paraná - PPAD/PUC-PR. Paraná, Brasil. E-mail: eliane.francisco@pucpr.br
} 


\section{INTRODUÇÃO}

Pesquisadores de marketing têm encontrado no stress uma possível causa para justificar mudanças nas atitudes dos consumidores à anúncios, produtos e marcas de forma geral, e especificamente na mudança por preferência de marca (Andreasen, 1984; Duhachek, 2005; Mathur, Moschis, \& Lee, 2006; Mehta \& Belk, 1991). Analisando comportamento, o autocontrole e em especial o controle de impulsos parece ser um mecanismo falho durante períodos de stress agudo. Mudanças no padrão alimentar, consumo de bebida alcoólica e valor monetário gasto em compras são comportamentos estudados já há algum tempo para analisar a impulsividade do consumidor (Giesen, Havermans, Nederkoorn, \& Jansen, 2012; O'Guinn \& Faber, 1989; Sayette, 1993; Williams \& Grisham, 2011).

O estímulo e avaliação do stress em ambiente laboratorial variam bastante conforme os sujeitos e objetivos da pesquisa. Protocolos de estímulos físicos, químicos, psicológicos e sociais são vistos na literatura, seja em modelos animais (Anderson et al., 1996; Pacak \& McCarty, 2000; Suárez, Paglini, \& Fernández, 1999) ou humanos (Flügge, 1996; Kirschbaum, Pirke, \& Hellhammer, 1993; Ottenweller, 2000; von Dawans, Kirschbaum, \& Heinrichs, 2011). Medidas subjetivas e de percepção já foram confrontadas com marcadores biológicos e parecem, de fato, corresponder à objetos distintos (Bradley, Miccoli, Escrig, \& Lang, 2008; Kreibig, 2010).

O cortisol é um hormônio liberado pelo organismo em situações de stress e sua medida salivar é capaz de registrar variações rápidas e de forma não invasiva (Pacak \& McCarty, 2000; J. Smyth et al., 1998; Smyth et al., 1997), parecendo esta ser uma forma viável e confiável para medir stress em protocolos experimentais com humanos. Neste contexto, os objetivos deste estudo foram: 1 . Validar o uso de um protocolo psicológico de indução de stress para participantes em grupo - GTSST (von Dawans et al., 2011) -, 2. Verificar o uso da medida de cortisol salivar como marcador biológico de stress viável para uso em pesquisas de comportamento do consumidor, e 3 . Confrontar o marcador biológico com medidas subjetivas e de percepção para o stress, a fim de verificar seus objetos e comportamentos medidos.

\section{A Definição de Stress}

Stress pode ser entendido por perspectivas biológica, psicológica e comportamental. A perspectiva biológica define o estado como a inabilidade do organismo em manter o equilíbrio dinâmico necessário à sobrevivência (Sterling \&
Eyer, 1988). As substâncias liberadas após o estímulo estressor, como neurotransmissores e hormônios, buscam o retorno deste equilíbrio dinâmico chamado alostasia (Levine, 2005). No prisma comportamental o stress é definido como qualquer demanda ambiental, social ou interna, que exija um ajuste nos padrões habituais de comportamento do indivíduo (Thoits, 1995). Estas demandas também podem gerar alterações no comportamento de consumo do indivíduo, levandoo a buscar novos padrões de conduta que objetivam, mesmo que de forma inconsciente, retomar o estado equilibrado do organismo (Mathur et al., 2006). O stress pode também moderar ansiedade e memória de trabalho, reduzindo a capacidade da memória de curto prazo em situações de stress agudo (Hoodac, Pulversa, Spadyb, Kliebensteina, \& Bachanda, 2015).

Biologicamente o stress pode ser entendido como uma sequência de eventos que começa com um estímulo (o estressor), gera uma reação no cérebro (percepção de stress) e que subsequentemente ativa sistemas fisiológicos no corpo (a resposta ao stress) (Dhabhar \& McEwen, 1997). O tipo de resposta gerada e sua intensidade são determinados pelo cérebro, em eixos específicos formados ainda durante a gestação e que podem ser influenciados pelo nível de stress materno no período (Weinstock, 2007). O sistema de stress envolve áreas corticais e subcorticais como o sistema límbico, do qual fazem parte a amígdala, que é relacionada ao processamento das emoções, o hipocampo, um dos responsáveis pela memória de informações aprendidas, o tronco encefálico, relacionado à ansiedade, e outras áreas responsáveis pelas tomadas de decisão (McEwen, 2007; Sapolsky, Romero, \& Munck, 2000). Aprendizado, memória e emoções são fatores que permeiam e influenciam de forma direta a relação de marcas com seus consumidores, tornando o estudo acadêmico das influências do stress fundamental para a compreensão desta relação. Da mesma forma, a tomada de decisão do consumidor é um objeto de estudo bastante importante dentro da disciplina de marketing, e considerar situações de stress e suas consequências amplia os conhecimentos gerados sobre o processo.

Durante episódios de stress agudo, a atividade simpática do Sistema Nervoso Autônomo (SNA) é ativada, e a parassimpática suprimida. O que significa dizer que o organismo torna-se mais ativo e preparado para lutar com uma ameaça ou fugir dela. Esta ativação leva à liberação na corrente sanguínea de cortisol, hormônio cuja produção envolve o Hipotálamo (região do encéfalo) e as glândulas Hipófise e Suprarrenais. Outros hormônios que não somente o cortisol, como por 
exemplo a adrenalina, além do neurotransmissor noradrenalina, também participam do desencadeamento dos mecanismos de resposta ao stress. Há um aumento na frequência dos batimentos cardíacos e da pressão arterial, o sistema imune é temporariamente suprimido e o metabolismo tornase catabólico (Mommersteeg, Heijnen, Kavelaars, \& van Doornen, 2006).

\section{Protocolos de Estímulo de Stress em Laboratório}

Estímulos estressores são classificados em diversas categorias, dispostos na literatura como físicos, psicológicos, sociais ou endócrinos; agudos ou crônicos, intermitentes ou contínuos, e ainda diferindo no grau de intensidade (Pacak, 2000).

Estudos experimentais sobre stress em ambiente laboratorial pressupõem formas de manipulação da variável, com uso de diversos protocolos. Estressores físicos são muito utilizados em modelos de pesquisa animais, e incluem calor (Pacak \& McCarty, 2000), frio (Taylor, 2000), imobilização (Jezova, Ochedalski, Glickman, Kiss, \& Aguilera, 1999), barulho (Ottenweller, 2000) e choque (Anderson et al., 1996). Estressores químicos, também utilizados em protocolos experimentais com animais, incluem a exposição ao éter (Suárez et al., 1999), substâncias tóxicas como o acetato de chumbo (Chang et al., 1997) ou formalina, uma solução aquosa do gás formol (Pacak \& McCarty, 2000). A privação da companhia materna ao nascimento também foi usada em modelos animais (King \& Edwards, 1999).

Estressores psicológicos, usados em pesquisas com seres humanos, como a exposição a ambientes desconhecidos e à novidade, afetam profundamente os processos emocionais e podem resultar em mudanças comportamentais, como ansiedade, medo ou frustração (Ottenweller, 2000). Como exemplos de estressores sociais estão as relações de hierarquia no trabalho e fatores como desemprego, separação conjugal além da perda de entes queridos (Flügge, 1996; Zelena, Haller, Halász, \& Makara, 1999).

Um dos protocolos de indução psicológica mais utilizados em estudos laboratoriais com humanos é o Trier Social Stress Test - TSST (Kirschbaum et al., 1993). O modelo é bastante eficiente em gerar situações de stress em laboratório (Armbruster et al., 2009; Gordis, Granger, Susman, \& Trickett, 2008; Hellhammer \& Schubert, 2012; Tomiyama, Dallman, \& Epel, 2011; Wolf, 2009), e seu protocolo geral envolve as tarefas de: 1 . Apresentar um briefing (informações preliminares sobre o a tarefa a ser executada) ao sujeito, permitindo alguns minutos de preparação, 2. Uma tarefa de entrevista gravada em áudio e vídeo onde o participante deve defender fortemente uma posição,
3. Uma tarefa aritmética de subtração mental bastante complexa e 4 . O debriefing (explicação detalhada do estudo). Uma versão adaptada para o TSST, chamada de Trier Social Stress Test for Groups - G-TSST - foi desenvolvida (von Dawans et al., 2011) para ser aplicada em grupos e o protocolo é bastante semelhante ao original, reduzindo os tempos individuais de entrevista e contagem numérica decrescente, mas buscando manter o nível de tensão entre os participantes.

Outros protocolos psicológicos descritos na literatura incluem o Stroop Test (Mitchell \& Epstein, 1996; Stroop, 1935) e alguns baseados em resoluções de atividades aritméticas diversas ou exposição de oratória em público (Gerra et al., 2001; Rutters, Nieuwenhuizen, Lemmens, Born, \& Westerterp-Plantenga, 2009; Torres, Turner, \& Nowson, 2010). Privação de sono (Altemus et al., 2001), frio (Lighthall, Gorlick, Schoeke, Frank, \& Mather, 2012) e barulho (Paris et al., 2010) foram vistos como protocolos físicos eficientes para gerar stress também em humanos. Por suas características psicológicas de indução, e por abranger mais de uma atividade potencialmente estressora, o G-TSST foi o protocolo utilizado neste estudo como estímulo de indução de stress nos participantes.

\section{Medidas de Stress em Pesquisas de Comportamento}

Em pesquisas acadêmicas é possível usar duas formas distintas de medidas para investigar ou confirmar estados de stress em humanos. Medidas subjetivas, ou questionários de percepção, são pautados em questionar o sujeito sobre a presença e o nível de stress que vivencia em determinado momento. De maneira distinta, marcadores biológicos não consideram a percepção do sujeito, mas sim as respostas que seu organismo gera. Hormônios e outras substâncias que estão circulantes no organismo, e os reflexos dessas substâncias como frequência cardíaca e respiratória, por exemplo, são medidas utilizadas para aferir a presença e intensidade do stress. É preciso que os pesquisadores compreendam as distinções para que possam escolher qual a de melhor aplicação no contexto que investigam.

\section{MEDIDAS SUBJETIVAS}

Uma maneira de avaliar a presença e intensidade do stress em humanos é por meio de protocolos subjetivos, como o uso de questionários (Fukui \& Yamashita, 2003) ou escalas visuais (Hellhammer \& Schubert, 2012). Os testes subjetivos tem por objetivo avaliar aspectos emocionais ou afetivos que parecem presentes em 
situações de stress, ou a percepção do sujeito sobre seu nível de stress.

A ansiedade associada ao stress é frequentemente mensurada através do State Trait Anxiety Inventory - STAI, um questionário que avalia dois tipos de ansiedade: o estado vivenciado naquele exato momento pelo respondente, e traços de ansiedade em sua personalidade, a escala é do tipo Likert, variando de 1 = nada, 2 = um pouco, $3=$ algum e 4 = muito (Spielberger \& Sydeman, 1994). $\mathrm{O}$ teste foi utilizado em diversas pesquisas que consideraram a ansiedade presente em situações de stress (Kang \& Fox, 2000; Muehlhan, Lueken, Wittchen, \& Kirschbaum, 2011; Newman, O'Connor, \& Conner, 2007; Paris et al., 2010; Rutters et al., 2009; Uchino, Holt-Lunstad, Uno, Betancourt, \& Garvey, 1999; von Dawans et al., 2011) e demonstrou relação positiva com medidas biológicas como pressão arterial, frequência cardíaca, níveis de cortisol e imunoglobulina A (Knight \& Rickard, 2001)

O comportamento das avaliações subjetivas de percepção de stress, ansiedade e insegurança emocional foram comparadas aos índices de cortisol salivar e frequência cardíaca durante a exposição ao protocolo TSST e todas as medidas foram significativamente maiores que as basais (Hellhammer \& Schubert, 2012). As flutuações em níveis de cortisol e testosterona em sujeitos submetidos a um protocolo que incluiu música e estímulos visuais de stress foram investigadas juntamente com um teste baseado no Profile of Mood States - POMS (McNair, Lorr, \& Droppleman, 1971) que solicitou ao participante um posicionamento frente a palavras que descreviam seu estado de humor, como "raiva" (Fukui \& Yamashita, 2003). O POMS é um questionário composto por 65 itens distribuídos em 6 fatores: tensão, depressão, raiva, fadiga, confusão e vigor que foram relacionadas como capazes de medir a resposta emocional decorrente do stress (Cella et al., 1987; Curran, 1995). Há adaptações para reduzir sua escala para 37 itens (Curran, 1995) bem como tradução da original para a língua portuguesa (Viana, Almeida, \& Santos, 2001)

Para investigar a influência do gênero e fase do ciclo menstrual sobre o stress, seis tipos de Escalas Visuais Analógicas foram utilizadas para medir stress gerado pelo TSST, que pediam ao indivíduo para classificar o quão estressante foi o estímulo (Kirschbaum, Kudielka, Gaab, Schommer, \& Hellhammer, 1999). Os níveis de cortisol salivar sofreram influência da flutuação hormonal, e as respostas seguiram o seguinte padrão: mulheres na fase lútea $=$ homens $>$ mulheres na fase folicular $=$ mulheres que usam contraceptivo hormonal. O questionário STAI e uma EVA de percepção de stress foram utilizados neste estudo por considerarem, além do stress compreendido pelo sujeito, a presença de ansiedade concomitante ao estado.

\section{MEDIDAS BIOLÓGICAS}

As consequências biológicas decorrentes do estímulo de stress, podem ser usadas como indicadores de sua presença e intensidade. Há diversas pesquisas utilizando medidas de hormônios como cortisol (Fukui \& Yamashita, 2003; Gerra et al., 2001; Hellhammer \& Schubert, 2012; Khalfa, Bella, Roy, Peretz, \& Lupien, 2003; Newman et al., 2007) testosterona (Fukui \& Yamashita, 2003), epinefrina, norepinefria, hormônio adrenocorticotrópico e prolactina (Gerra et al., 2001; Peters et al., 1998) ou anticorpos como a imunoglobulina A (McCraty, Atkinson, Rein, \& Watkins, 1996), para investigar a presença de stress em humanos. Outras respostas do aumento da atividade simpática como a frequência cardíaca e a pressão arterial também são vistas enquanto indicadores de stress (Knight \& Rickard, 2001; Peters et al., 1998; Uchino et al., 1999).

O cortisol é excretado na urina, no plasma sanguíneo e na saliva, e sua dosagem salivar é um ótimo indicador da quantidade ativa no organismo (Dhabhar \& McEwen, 1997; Keller, 2012; Levine, 2005). O procedimento metodológico pelo qual as medidas salivares de cortisol podem ser utilizadas como marcadores para o stress em pesquisas clínicas foi definido em 1993 (Kirschbaum et al., 1993) e replicado por diversas vezes com efetividade (Kudielka, Hellhammer, \& Kirschbaum, 2000; Smyth et al., 1997; Smyth et al., 1998; Steptoe, Cropley, Griffith, \& Kirschbaum, 2000), embora nunca em pesquisas de marketing. Além de liberado em grande quantidade durante episódios de stress, o cortisol possui a função de marcador cronobiológico no organismo humano, com expressões diárias seguindo um ciclo diurno. Picos na expressão de cortisol acontecem logo ao despertar e seguem em decréscimo até valores mínimos durante a noite (Zilioli, Imami, \& Slatcher, 2015). Em sujeitos considerados "noturnos", ou que se sentem mais dispostos no final do dia, os picos de cortisol acontecem um pouco mais tarde que em sujeitos "diurnos" (Bailey \& Heitkemper, 1991).

A avaliação do nível salivar de cortisol entre homens e mulheres expostos a diversas situações estressantes aponta que entre participantes saudáveis - sem stress crônico diagnosticado -, os homens apresentam níveis basais maiores. A resposta feminina ao stress agudo gera níveis do hormônio iguais ou maiores que a masculina. Pessoas viciadas em jogos têm respostas mais exacerbadas aos estímulos de stress da partida se comparadas à de quem participa de jogos por 
recreação, e esses efeitos são mais proeminentes entre os homens do que em mulheres (Paris et al., 2010). Diferenças fisiológicas nas áreas neurologicamente ativas durante episódios de stress entre homens e mulheres também foram encontradas com a análise de imagens de ressonância magnética funcional - fMRI (Lighthall et al., 2012).

A dosagem de cortisol salivar oferece múltiplas vantagens sob as dosagens em soro ou plasma. As coletas das amostras são simples, não invasivas e seu custo é baixo, dispensando a necessidade de um ambiente preparado para tal (Read, Walker, Wilson, \& Griffiths, 1990). A produção de cortisol é intimamente ligada ao ciclo circadiano, com níveis de pico máximo pela manhã, por volta de $8 \mathrm{~h}$ e mínimo a noite, perto das $24 \mathrm{~h}$ (Krieger, 1975). O autor ainda cita que aumentos de cortisol podem acontecer independentemente do ritmo circadiano em resposta a stress físico e psicológico e os valores de referência devem ser definidos por cada laboratório, conforme seu protocolo de avaliação. A medida de cortisol salivar foi utilizada neste estudo como marcador biológico para o stress, comparando-se os níveis pré-pós aplicação do estímulo G-TSST.

\section{METODOLOGIA}

Este estudo de características experimentais possui design between subjects, com manipulação da variável stress. Sua realização foi aprovada pelo Comitê de Ética em Pesquisa da Instituição de Ensino onde foi conduzido, no Parecer Consubstanciado $\mathrm{n}^{\mathrm{o}}$ 157.853, em 28/09/2012. A coleta de dados aconteceu nos meses de outubro a dezembro de 2012.

\section{Design Experimental}

A amostra foi formada por conveniência, composta por 27 mulheres e 23 homens saudáveis (sem diagnóstico clínico de stress, segundo autorrelato), divididos em dois grupos - controle e experimental. Não participaram da amostra sujeitos menores de 18 anos, fumantes, usuários de drogas ansiolíticas ou calmantes, que tivessem ingerido bebida alcoólica e/ou passado por privação de sono nas 24 horas antecedentes ao dia da coleta de dados, fatores que poderiam influenciar seus níveis de cortisol circulantes, conforme fizeram, dentre outros, Bailey e Heitkemper (1991). Além disso, os participantes foram orientados a preencher um relato e subsequente avaliação de possíveis causadores de stress vividos nas 24 horas antecedentes ao estudo. Os participantes que relatassem ter vivido algum momento significativo de stress anterior ao estudo seriam excluídos da análise, o que não aconteceu porque nenhum deles relatou episódio que considerasse significativo.

O stress foi mensurado biologicamente pela variação entre os níveis de cortisol salivar circulantes no sujeito nos momentos pré e pós estímulo. A coleta de saliva aconteceu em tubos Salivete ${ }^{\circledR}$ próprios para tal, de acordo com o protocolo do laboratório Frishmann Aisengart, responsável pelas análises. De acordo com o protocolo, para cada round de coleta os participantes receberam um tubo Salivette ${ }^{\circledR}$ que continha um pedaço de algodão em seu interior, deveriam colocar o algodão debaixo da língua por 2-3 minutos e então cuspi-lo dentro do tubo e fecha-lo, sempre sozinhos. O responsável pela coleta das amostras, fisioterapeuta, vestia luvas descartáveis e armazenava os tubos em um compartimento térmico adequado. Ao final de cada dia de experimento as amostras foram imediatamente conduzidas ao laboratório, centrifugadas e congeladas a $-20 \mathrm{C}$, para posterior análise de toda a coleta por eletroquimioluminescencia. Para aferir subjetivamente o stress foram utilizados uma Escala Visual Analógica - EVA - de percepção de stress e o State Trait Anxiety Inventory - STAI - (Paris et al., 2010; Hellhammer \& Schubert, 2012).

Para a manipulação do stress foi utilizado o protocolo G-TSST com uma pequena alteração: a entrevista de emprego foi substituída por entrevista para bolsa de intercâmbio estudantil, ou para a possibilidade de ganhar 2 pontos na média final de uma disciplina determinada pelo aluno, buscando maior envolvimento do sujeito com o ambiente simulado proposto. O contexto foi testado previamente com três sujeitos e se mostrou efetivo no engajamento e na mimetização de um cenário real, conforme relato dos participantes. Também foram comparados os valores finais e iniciais de cortisol salivar, STAI e EVA destes sujeitos e embora não haja dados suficientes para análises estatísticas, em todos o comportamento foi como esperado: valores finais maiores que os iniciais. Os dados coletados durante o experimento foram analisados com uso do software SPSS através dos testes de diferença de média - teste t-Student - e de Correlação de Pearson entre as medidas.

\section{Procedimento Experimental}

$\mathrm{Na}$ chegada ao local indicado, os voluntários - alunos de primeiro período em uma universidade privada - foram informados de que participariam de uma pesquisa sobre comunicação, que deveriam avaliar dois anúncios publicitários e responder algumas perguntas sobre o anúncio e a marca anunciada. Receberam o Termo de Consentimento Livre e Esclarecido, tiveram o tempo necessário para ler e tirarem dúvidas. 
O experimento teve início com a primeira coleta de amostra de saliva as $8 \mathrm{~h}$, horário do pico máximo de cortisol em humanos saudáveis. $\mathrm{Na}$ sequência todos os participantes responderam aos questionários STAI e EVA. Os grupos foram divididos aleatoriamente e metade dos participantes foi conduzida à uma sala anexa, para que recebesse o estímulo de stress, enquanto os demais aguardaram com revistas e livros para distração, o que caracteriza a manipulação do tipo between subjects. A leitura oferecida ao grupo controle abordava esportes em geral, guias gastronômicos ou moda, buscando minimizar um possível efeito estressor sobre os participantes.

O estímulo de stress transcorreu da seguinte forma: os sujeitos do grupo experimental, em número máximo de seis, foram conduzidos a uma sala de aproximadamente $20 \mathrm{~m}^{2}$, contendo apenas os móveis necessários para o estudo e uma grande janela, preparada com duas câmeras filmadoras que simulavam uma gravação, 6 cadeiras separadas por biombos e uma mesa para dois avaliadores externos, conforme a Figura 1. Ao se instalarem em seus lugares, os participantes foram informados de que a entrevista seria conduzida por dois avaliadores externos e que as imagens de suas entrevistas seriam analisadas posteriormente para a escolha de dois vencedores: um para a bolsa de intercâmbio, e outro para o bônus de 2 pontos, conforme suas preferências. Cada participante teve 2 minutos para justificar porque seria merecedor de ganhar o prêmio e quando encerraram suas falas antes do término do tempo, foram conduzidas interferências da seguinte forma:

A. Os avaliadores informavam o tempo restante à disposição do aluno para sua defesa.

B. Os avaliadores questionavam o aluno sobre: i) "você acha que é o melhor aluno para ganhar o prêmio?", ii) "por que você acha que é o melhor?”, iii) “quais são seus planos para depois que terminar a graduação?”.

C. Os avaliadores permaneciam em silêncio até o final do tempo de 2 minutos.

Concluídas as entrevistas com todos os participantes, os avaliadores informaram que teriam ainda uma segunda tarefa, desta vez aritmética, para resolver: deveriam subtrair mentalmente o número 16 de um número inicial determinado, teriam 90 segundos para efetuarem as subtrações e a cada resultado errado reiniciariam o procedimento. Os avaliadores acompanhavam os resultados com uma calculadora, a cada erro foi pedido ao participante que reiniciasse sua subtração sequencial. Os números iniciais fornecidos aos participantes foram: 5346, 7286, 2256, 8436, 4926 e 5226. A escolha pelo número 16 a ser subtraído partiu do protocolo original, que também usou este valor. O protocolo não informa os números iniciais e apenas apresenta um exemplo de 4 dígitos, foram então definidos os valores iniciais aleatoriamente com o objetivo de tornar a primeira subtração relativamente fácil (todos terminam em 6). As entrevistas duraram em média 30 minutos.

Ao final do protocolo os participantes foram conduzidos novamente à primeira sala do estudo, juntando-se aos demais. A segunda amostra de saliva foi coletada, os sujeitos responderam novamente ao STAI e à EVA e avaliaram por meio de questionários de atitude os dois anúncios que receberam. Em média cada dia de coleta teve duração de 90 minutos. O debriefing aconteceu após o término de toda a coleta, para evitar contaminação da amostra. Foram enviados e-mails a todos os participantes explicando detalhadamente os objetivos da pesquisa e da manipulação, nenhum participante respondeu aos pesquisadores. Dois participantes foram contactados pessoalmente e demonstraram ter entendido as razões do cenário fictício.

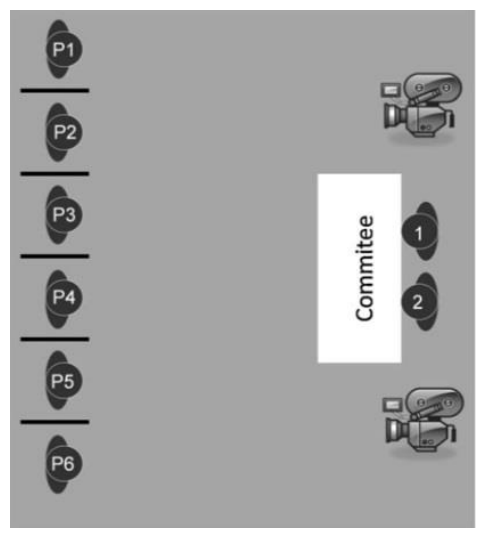

Figura 1 - Ilustração da estrutura de aplicação do G-TSST

Fonte: von Dawans et al., 2011 


\section{RESULTADOS}

A amostra foi composta por 50 estudantes, sendo 25 para o grupo controle e 25 para o grupo experimental, com média de idade de 20,56 anos, dos quais $27(54 \%)$ eram mulheres e $23(46 \%)$ homens. Não foi necessária, por critério que se justificasse no estudo, a exclusão de nenhum participante durante/após a análise dos dados coletados, mantendo-se os 50 respondentes como válidos.

A análise de variáveis dependentes iniciou com a observação das distribuições dos dados. Quatro variáveis apresentaram distribuição igual à normal (STAI1, STAI 2, Cortisol1 e DeltaSTAI), três variáveis apresentaram distribuição próxima da normal, com valores de assimetria e curtose menores que 1 (EVA1, EVA2 e DeltaEVA) e apenas duas variáveis apresentaram curvas diferentes da normal, com valores de assimetria e curtose maiores que 1 (Cortisol2 e DeltaCortisol). Em casos como este e em alguns outros casos onde há violação parcial dos pressupostos de normalidade, Marôco (2011) e Malhotra (2001) defendem que ANOVA e t-Student são testes robustos e podem ser aplicados ainda que não haja normalidade completa, o que resulta em extensão do teorema do limite central. Por se tratar de duas referencias bastante sólidas na área das ciências sociais aplicadas e em especial do marketing, optou-se por utilizar os mesmos em detrimento de testes não paramétricos.

Os valores iniciais e finais de cortisol salivar, STAI e EVA apresentados pelos sujeitos do grupo controle e experimental foram comparados por meio do teste t-Student pareado. Os níveis de cortisol salivar são apresentados em microgramas por decilitro de saliva $-\mu / \mathrm{dL}-$, o valor mínimo passível de medida é $0 \mu / \mathrm{dL}$, e não há limite máximo estabelecido, uma vez que se trata da resposta biológica do indivíduo ao estímulo. O STAI é um questionário formado por 20 itens ancorados em 1 (mínimo) e 4 (máximo), seu resultado mínimo possível é 20, e o máximo 80. A EVA de stress percebido utilizada variou de 0 (mínimo) a 10 (máximo). Os valores do teste t-Student, bem como o tamanho do efeito, medido através do indicador Cohen's d, estão descritos na Tabela 1 .

Tabela 1 - Média, Desvio Padrão e Teste T pareado entre as médias das variáveis de controle do stress, conforme o grupo amostral.

\begin{tabular}{lcccccc}
\hline \multicolumn{1}{c}{ Grupo } & Medida & Dif. Média & Desvio Padrão & $\mathbf{t}$ & Sig(2-tailed) & Cohen's d \\
\hline Controle & Cort.2-Cort.1 &,- 1044 &, 10767 & 4,848 &, 000 & 0.3465 \\
Controle & STAI2-STAI1 & 1,680 & 7,931 & $-1,059$ &, 300 & -0.2276 \\
Controle & EVA2-EVA1 &, 200 & 1,607 &,- 622 &, 540 & -0.0968 \\
Experimental & Cort.2-Cort.1 &, 0340 &, 2627 &,- 647 &, 524 & -0.1639 \\
Experimental & STAI2-STAI1 & 8,600 & 8,597 & $-5,001$ &, 000 & -1.4433 \\
Experimental & EVA2-EVA1 & 1,220 & 1,696 & $-3,597$ &, 001 & -0.5591 \\
\hline
\end{tabular}

Nota: Coleta 1: Pré-estímulo, coleta 2: Pós-estímulo. $\mathrm{N}=25$ por grupo.

Fonte: análise de dados do estudo.

O teste indicou diferenças significativas no grupo experimental entre os momentos pré/pósestímulo para as variáveis STAI e EVA, com aumento nos valores da segunda resposta dos participantes, o que indica maior percepção de stress pelos participantes do grupo experimental. Embora os valores da segunda medida tenham aumentado também no grupo controle a diferença não se mostrou significativa. Os efeitos foram mais percebidos para a variável STAI que para a EVA maiores índices de Cohens'd.
Para os valores de cortisol, houve redução significativa dos níveis circulantes nos sujeitos do grupo controle, o que era esperado considerando o horário das medidas (Balbo, Leproult, \& Van Cauter, 2010). O grupo experimental apresentou índices maiores de cortisol salivar pós-estímulo, porém não significativamente diferentes se comparados aos iniciais. Os gráficos 1,2 e 3 ilustram o comportamento das variáveis. 


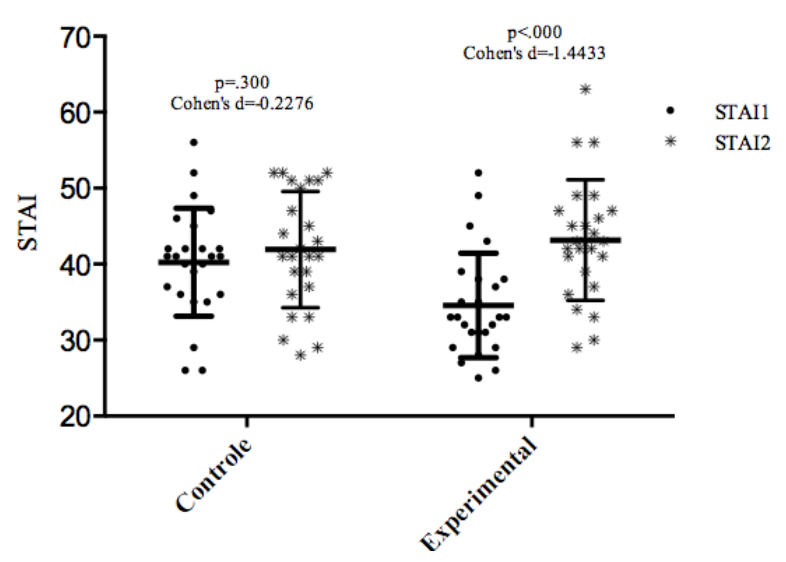

Gráfico 1 - Valores de STAI pré/pós estímulo para grupo controle e experimental Nota: $\mathrm{N}=50$ por grupo.

Fonte: análise de dados do estudo.

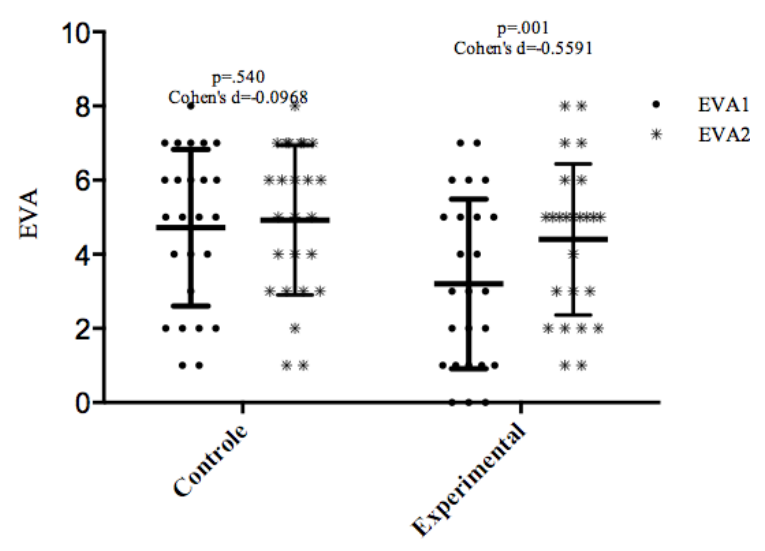

Gráfico 2 - Valores de EVA pré/pós estímulo para grupo controle e experimental Nota: $\mathrm{N}=50$ por grupo.

Fonte: análise de dados do estudo.

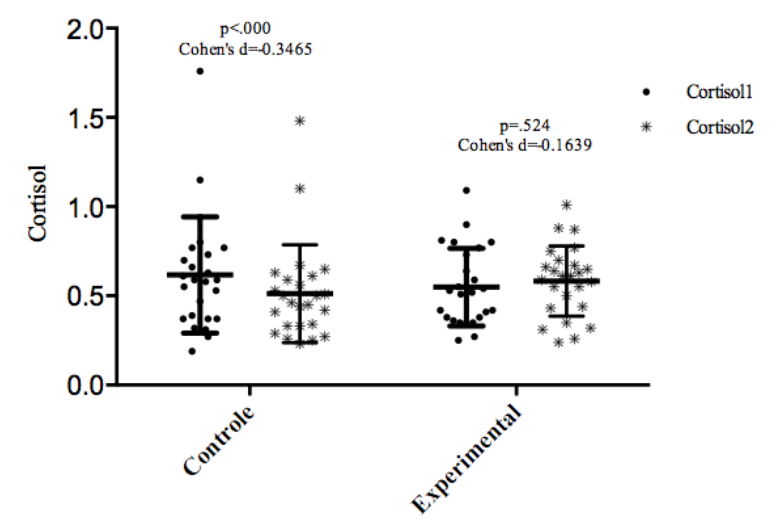

Gráfico 3 - Valores de Cortisol pré/pós estímulo para grupo controle e experimental Nota: $\mathrm{N}=50$ por grupo.

Fonte: análise de dados do estudo. 
Caso não tivessem passado pelo protocolo de stress, todos os sujeitos deveriam ter se comportado como o grupo controle: com redução da segunda medida de cortisol, dado o horário das coletas. Uma vez que os valores se comportaram de maneira contrária - com aumento na segunda medida -, foram comparadas então por meio do teste t-Student para amostras independentes, os valores de cortisol em delta (final - inicial), entre os grupos, o que evidenciou a discrepância entre as medidas e comprovou a eficácia do protocolo utilizado $\left(\mathrm{M}_{\text {CONTROLE }}=-, 1044, \mathrm{M}_{\text {EXPERIMENTAL }}=0,0340, \mathrm{t}=-\right.$ $2,437, \mathrm{p}=, 019)$. Foram investigados também as diferenças de médias dos valores em delta para as variáveis STAI $\quad\left(\mathrm{M}_{\mathrm{CONTROLE}}=1,68 \quad\right.$, $\left.\mathrm{M}_{\text {EXPERIMENTAL }}=8,60, \mathrm{t}=-2,958, \mathrm{p}=, 034\right)$ e EVA

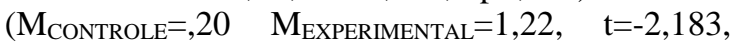
$\mathrm{p}=, 005$ ), a fim de comparar seus resultados entre grupos. As três variáveis apresentaram comportamentos significativamente diferentes entre os grupos, com efeito sutilmente maior para a medida de STAI. Os Gráficos 4, 5 e 6 ilustram as diferenças. O protocolo G-TSST foi capaz de anular o efeito de redução no cortisol, esperado para sujeitos não estressados por conta do horário, e ainda aumentar os índices, tornando as variações significativamente distintas entre os grupos.

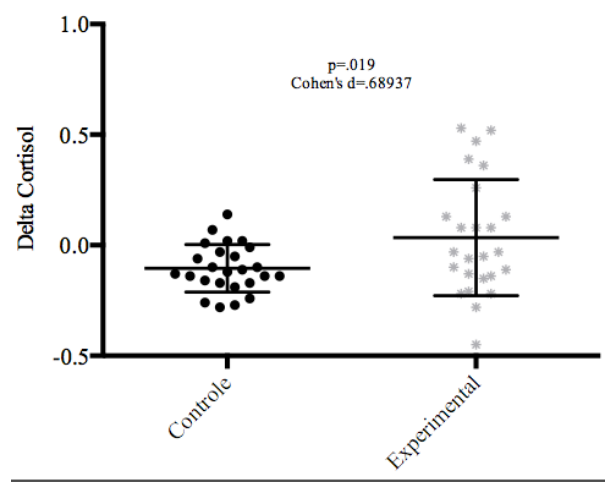

Gráfico 4 - Valores em delta para as medidas de Cortisol pré/pós estímulo para grupo controle e experimental Nota: $\mathrm{N}=50$ por grupo.

Fonte: análise de dados do estudo

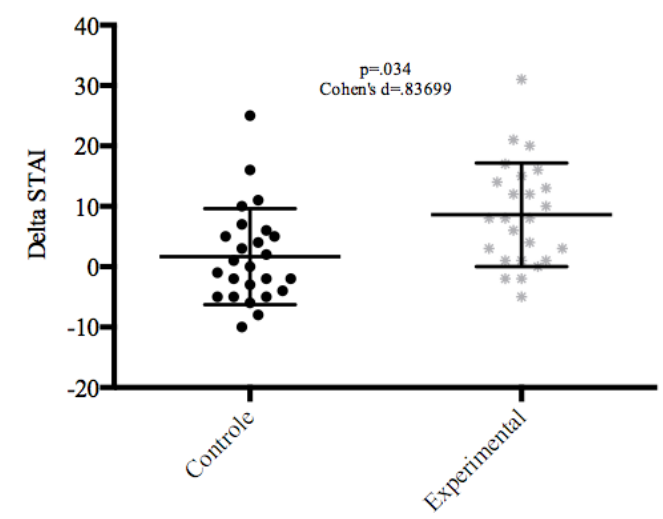

Gráfico 5 - Valores em delta para as medidas de STAI pré/pós estímulo para grupo controle e experimental

Nota: $\mathrm{N}=50$ por grupo.

Fonte: análise de dados do estudo. 


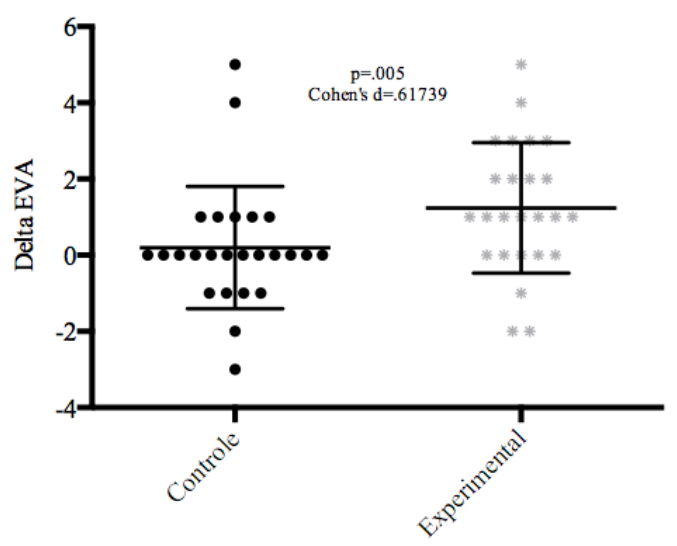

Gráfico 6 - Valores em delta para as medidas de EVA pré/pós estímulo para grupo controle e experimental

Nota: $\mathrm{N}=50$ por grupo.

Fonte: análise de dados do estudo.

A análise do comportamento das variáveis de controle do stress conforme o gênero apontou diferenças marginalmente significativas apenas para os valores de cortisol. As mulheres apresentaram níveis basais de cortisol superiores aos homens $\left(\mathrm{M}_{\text {MULHERES }}=, 6459, \mathrm{M}_{\text {HOMENS }}=, 5087, \mathrm{p}=, 080, \mathrm{n}=50\right)$, sem diferenças na segunda medida de cortisol. A diferença entre o valor final e inicial de cortisol foi distinta entre os gêneros apenas para o grupo controle, com variações mais intensas para as mulheres que para os homens $\left(\mathrm{M}_{\text {MULHERES }}=-, 1182\right.$, $\left.\mathrm{M}_{\text {HOMENS }}=-, 0750, \mathrm{p}=, 019, \mathrm{n}=25\right)$. O que significa dizer que as mulheres iniciaram o estudo com níveis mais altos de cortisol que os homens, e que em situação de não stress, reduziram esses níveis com o passar do tempo com mais intensidade. A maior intensidade de resposta ao stress entre as mulheres, descrita na literatura (Paris et al., 2010) não foi verificada nos participantes.

Ainda, buscando evidenciar a relação entre as variáveis subjetivas e a medida biológica, o teste de Correlação de Pearson identificou forte correlação entre as variáveis subjetivas no grupo controle, e alguma correlação no grupo experimental, mas ausência de correlação destas com a variável biológica em qualquer dos grupos, como apresentado na Tabela 2.

Tabela 2 - Correlação entre as variáveis STAI, EVA e Cortisol Salivar

\begin{tabular}{lcccccc}
\hline & & Controle & & \multicolumn{3}{c}{ Experimental } \\
\cline { 2 - 7 } STAI1 & STAI1 & EVA1 & CORT1 & STAI1 & EVA1 & CORT1 \\
\cline { 2 - 7 } EVA1 & 1 &, $692^{* *}$ &, 342 & 1 &, 302 &, 089 \\
CORT1 & & 1 &, 161 & & 1 &,- 104 \\
& & & 1 & & & 1 \\
STAI2 & STAI2 & EVA2 & CORT & STAI2 & EVA2 & CORT2 \\
EVA2 & 1 &, $635^{* *}$ &, 181 & 1 &, $411^{*}$ &, 154 \\
CORT2 & & 1 &,- 011 & & 1 &,- 237 \\
\hline
\end{tabular}

Nota: $* * \operatorname{sig}<0,01, * \operatorname{sig}<0,05 . \mathrm{N}=25$ por grupo.

Fonte: análise de dados do estudo.

Tomadas em conjunto, todas as análises indicam que os membros do grupo experimental estavam de fato estressados, e que não houve indução de stress nos sujeitos do grupo controle. Além disso, a correlação entre as medidas subjetivas, e a ausência de correlação entre elas e a medida biológica, além do comportamento observado nos escores subjetivos e biológicos, evidencia que são mensurações de objetos distintos. Enquanto as escalas subjetivas são reflexos da percepção do sujeito sobre seu nível de stress, a medida biológica demonstra o que acontece com seu organismo naquele momento. E sendo distintas, as medidas não são substituíveis. 


\section{DISCUSSÃO}

Protocolos extensos de pesquisa, com manipulação de diversas variáveis e interferências de controle podem fazer com que o engajamento do participante diminua no decorrer do tempo pelo cansaço, e as últimas atividades não sejam executadas com a mesma aliciação despendida nas primeiras. Via de regra as últimas variáveis são comprometidas em situações como esta, e encontrar o melhor caminho para burlar o desinteresse do sujeito é um grande desafio aos pesquisadores.

Um potencial causador de cansaço nos participantes é a aplicação de vários questionários consecutivos, que embora apresentem a grande vantagem de investigar múltiplas variáveis, demandam tempo, disposição e participação ativa do respondente, sob pena de não serem fiéis à realidade (Strahler et. al, 2010; Muehlhan et. al, 2011; Von Dawans, Kirschbaum \& Heinrichs, 2011). O uso de escalas simples pode ser uma alternativa à protocolos extensos, mas como única medida deixa a desejar na profundidade da investigação e confiabilidade dos resultados. Caso seja a opção tida como mais viável, a utilização de escalas simples combinada com outras medidas pode aumentar a confiança nos resultados (Rutters et al., 2009).

Uma alternativa que vem ganhando espaço nas pesquisas em comportamento do consumidor é o uso de marcadores biológicos para a confirmação de estados afetivos e/ou emocionais. Frequência cardíaca, respiratória, ondas elétricas musculares ou neurais, hormônios, anticorpos, movimentos oculares e outras funções biológicas são relacionadas a estados específicos do corpo humano, e podem servir como escores de confirmação de ativação do sistema autonômico, fato que independe da percepção do sujeito (Bradley et al., 2008; Kreibig, 2010). Protocolos de avaliação mistos com medidas subjetivas e de percepção podem ser uma boa alternativa para oferecer dados confiáveis e completos aos pesquisadores. A avaliação subjetiva através do Multidimensional Mood Questionare MMQ, versão em inglês do MDBF (Steyer et. al, 1994) em conjunto com medidas biológicas de cortisol e alfa-amilase salivares (Muehlhan et al., 2011) ou entre medidas subjetivas como o STAI, questionários de percepção simples como EVA, e marcadores biológicos como o cortisol salivar e a frequência cardíaca (Hellhammer \& Schubert, 2012) foram eficientes nesta proposta.

Um dos objetivos deste estudo foi validar o protocolo psicológico de indução de stress G-TSST para estudos de marketing, além de comparar em um mesmo sujeito suas respostas subjetivas, de percepção e biológicas consequentes ao estímulo. A análise dos resultados de cortisol salivar tomados em conjunto com as medidas de STAI e EVA, permite afirmar que o protocolo utilizado para o estímulo de stress agudo nos participantes foi efetivo no que se propôs. O formato adaptado de G-TSST (von Dawans et al., 2011) utilizado, gerou de fato o estado de stress agudo nos sujeitos do grupo experimental.

A medida de cortisol salivar foi escolhida como marcador biológico a ser testado por ser um hormônio liberado pelo organismo humano em estado de stress agudo (Ribeiro, Kennedy, Smith, Stohler, \& Zubieta, 2005; Sapolsky et al., 2000) e permitir a aferição de variações pequenas em um curto período de tempo (Hellhammer \& Schubert, 2012; Paris et al., 2010), além da apresentação viável de uma medida salivar, e portanto não invasiva (Smyth et al., 1998). O questionário STAI foi utilizado por ser uma medida subjetiva e pela característica do protocolo, que poderia envolver também estado de ansiedade; a EVA se fez presente por ser uma avaliação de percepção objetiva e rápida.

Ao confrontar o comportamento das três medidas, nota-se um direcionamento divergente entre elas. Enquanto o nível de cortisol salivar diminuiu significativamente seus valores entre o início e o final do estudo para o grupo controle - o que era esperado pelo período de repouso dos participantes e pelo horário das coletas (Krieger, 1975) -, os escores das medidas de percepção aumentaram neste grupo e período, ainda que de maneira não significativa. Os sujeitos então, fisiologicamente reduziram seu nível de stress, mas se perceberam mais estressados e ansiosos, indicando que as medidas podem não ser substituíveis ou idênticas em termos de objeto medido. Cabe neste momento a reflexão sobre o que de fato interessa medir em cada pesquisa, e qual o melhor método avaliativo. Os dados inspiram cautela na escolha de variáveis de medida em estudos sobre stress. Será a percepção de stress diferente do stress fisiológico? Qual destas variáveis é mais interessante para responder determinado problema de pesquisa? Comportamento parecido foi encontrado em um estudo recente que investigou a relação entre cortisol capilar (uma forma de medida crônica do stress), stress percebido e diversas outras variáveis como clima com treinamento militar de alta intensidade em recrutas do exército suíço, e verificou que os sujeitos relataram aumento de stress percebido em um intervalo de 10 semanas, mas que este aumento não foi observado na medida biológica; além disso, temperatura, humidade e educação foram, sim, variáveis correlacionadas com os valores de cortisol capilar (Boesch et al., 2015).

Ainda, aqui, a medida biológica foi bastante eficaz em representar o estado vivido pelo sujeito, o que a torna uma alternativa interessante ao problema de protocolos extensos. A resposta biológica não passa pelos crivos racionais, ou supraliminares do 
participante, e portanto não deve sofrer interferência imediata do seu desinteresse. Com a ampliação e facilidade de acesso a essas medidas, elas deveriam se tornar uma alternativa significativa para os pesquisadores de comportamento de consumo, reiterando sua distinção em termos de objeto medido.

Este estudo também incrementa a discussão do uso de cortisol como marcador para stress em pesquisas de comportamento destacando possíveis variáveis de influência na expressão e regulação do cortisol em humanos, e sugerindo mais investigações sobre suas influências nas variáveis de marketing. Pessoas com altos níveis socioeconômicos e de satisfação com a vida apresentam reduções íngremes nos níveis de cortisol após o pico matutino, o que significa que sofrem menos os efeitos negativos do cortisol e do stress ao longo do dia, em comparação a pessoas com baixos níveis socioeconômicos ou baixa satisfação com a vida; a satisfação com a vida, ainda, pode atenuar o efeito negativo do baixo status socioeconômico sobre o stress (Zilioli et al., 2015). É possível que a satisfação com a vida também interfira em avaliações de marcas, anúncios, intenção de compra e outras variáveis de marketing, e esta é uma faceta que precisa ser investigada em pesquisas futuras de marketing. O pico de cortisol ao despertar também é influenciado por stress crônico, fadiga, stress pós-traumático e maus tratos na infância (Mello et al., 2015), outras variáveis que devem ser investigadas em pesquisas futuras e que podem enriquecer as teorias de comportamento do consumidor.

Por fim, destaca-se que os pesquisadores dispostos a usar marcadores biológicos em suas pesquisas devem conhecer profundamente as limitações inerentes ao seu uso, a fim de evitar vieses nos resultados. O organismo humano é impactado por diversos fatores endógenos e exógenos ao sujeito, e a redução dessas variáveis nas coletas só acontecerá mediante conhecimento prévio e profundo do instrumento de medida. Neste estudo, filtros inseridos na escolha dos sujeitos e outros de controle durante o estudo buscaram evitar a influência de remédios, estados emocionais e outras condições (como ser fumante) que poderiam impactar na expressão de cortisol medida. A distribuição aleatória dos sujeitos entre grupos, por sua vez, buscou minimizar o alcance de variáveis incontroláveis mas conhecidas, como hábitos de sono, sobre os resultados.

\section{CONCLUSÕES, LIMITAÇÕES E SUGESTÕES PARA PESQUISAS FUTURAS}

Foram validados neste estudo a medida de cortisol salivar como marcador biológico para stress, e um protocolo de estímulo de stress psicológico versão adaptada do G-TSST (von Dawans et al., 2011) - para uso em pesquisas empíricas. Os valores de cortisol salivar foram confrontados com outros de avaliações subjetivas de percepção de stress STAI e EVA -, e os resultados sugerem que não são indicadores substituíveis entre si.

A medida biológica do cortisol salivar é, portanto, confiável em sua utilização como marcador de stress para pesquisas de comportamento, e deve ser escolhida em casos de protocolos extensos ou quando há necessidade de confirmação de estado de stress nos participantes. As medidas de percepção devem ser consideradas quando a investigação se interessa justamente pela percepção do sujeito, como em casos de medidas de autoeficácia, autocontrole percebido ou outros cenários que envolvam autoavaliações.

A utilização de marcadores biológicos decorrentes de estados psicológicos e/ou emocionais em pesquisas de comportamento do consumidor é uma metodologia pouco explorada, e ao passo que a facilidade de acesso a tais medidas aumenta, deverse-ia aumentar também consideração sobre a presença delas em nossas pesquisas. Ainda, o desenvolvimento e aplicação de novos protocolos de estímulo de stress, que demandem menor tempo de preparação e aplicação simultânea a mais participantes é uma lacuna a ser preenchida pelos estudiosos da área.

Uma das limitações encontradas com o uso de marcadores biológicos, e em especial de medidas de stress, é a dificuldade de certeza sobre o estado diagnóstico clínico dos participantes previamente ao estudo. Não houve diagnóstico médico sobre a ausência de stress nos participantes, como não houve para diversas outras patologias que poderiam influenciar a expressão de cortisol salivar. A distribuição aleatória dos sujeitos entre os grupos buscou minimizar a influência destas e de outras variáveis sobre os resultados encontrados.

\section{REFERÊNCIAS}

Altemus, M., Rao, B., Dhabhar, F. S., Ding, W., \& Granstein, R. D. (2001). Stress-induced changes in skin barrier function in healthy women. The Journal of Investigative Dermatology, 117(2), 30917.

Anderson, S. M., Saviolakis, G. a, Bauman, R. a, Chu, K. Y., Ghosh, S., \& Kant, G. J. (1996). Effects of chronic stress on food acquisition, plasma hormones, and the estrous cycle of female rats. Physiology \& Behavior, 60(1), 325-329. 
Andreasen, A. R. (1984). Life status changes and changes in consumer preferences and satisfaction. Journal of Consumer Research, 11(3), 784-794.

Armbruster, D., Mueller, a, Moser, D. a, Lesch, K. P., Brocke, B., \& Kirschbaum, C. (2009). Interaction effect of D4 dopamine receptor gene and serotonin transporter promoter polymorphism on the cortisol stress response. Behavioral Neuroscience, 123(6), 1288-95.

Bailey, S. L., \& Heitkemper, M. M. (1991). Morningness-eveningness and early-morning salivary cortisol levels. Biological Psychology, 32(2-3), 181-192.

Balbo, M., Leproult, R., \& Van Cauter, E. (2010). Impact of sleep and its disturbances on hypothalamo-pituitary-adrenal axis activity. International Journal of Endocrinology, 2010, 759234.

Boesch, M., Sefidan, S., Annen, H., Ehlert, U., Roos, L., Van Uum, S., ... La Marca, R. (2015). Hair cortisol concentration is unaffected by basic military training, but related to sociodemographic and environmental factors. Stress: The International Journal on the Biology of Stress, 18(1), 35-41.

Bradley, M. M., Miccoli, L., Escrig, M. a, \& Lang, P. J. (2008). The pupil as a measure of emotional arousal and autonomic activation.

Psychophysiology, 45(4), 602-7.

Cella, D. F., Jacobsen, P. B., Orav, E. J., Holland, J. C., Silberfarb, P. M., \& Rafla, S. (1987). A brief POMS measure of distress for cancer patients. Journal of Chronic Diseases, 40(10), 939-42.

Chang, H. R., Chen, S. S., Tsao, D. a, Cheng, J. T., Ho, C. K., \& Yu, H. S. (1997). Change of cardiac beta-adrenoceptors in lead-exposed rats. Toxicology, 123(1-2), 27-32.

Curran, S. (1995). Short Form of the Profile of Mood States (POMS-SF): Psychometric information. Psychological Assessment, 7(1), 8083.

Dhabhar, F. S., \& McEwen, B. S. (1997). Acute stress enhances while chronic stress suppresses cellmediated immunity in vivo: a potential role for leukocyte trafficking. Brain, Behavior, and Immunity, 11(4), 286-306.
Duhachek, A. (2005). Coping : A Multidimensional , Hierarchical Framework of Responses to Stressful Consumption Episodes, 32(June).

Flügge, G. (1996). Alterations in the central nervous $\alpha 2$-adrenoceptor system under chronic psychosocial stress. Neuroscience, 75(1), 187-196.

Fukui, H., \& Yamashita, M. (2003). the effects of music and visual stress on testosterone and cortisol in men and women. Neuro Endocrinology Letters, 24(3/4), 173-180.

Gerra, G., Zaimovic, a, Mascetti, G. G., Gardini, S., Zambelli, U., Timpano, M., ... Brambilla, F. (2001). Neuroendocrine responses to experimentally-induced psychological stress in healthy humans. Psychoneuroendocrinology, 26(1), 91-107.

Giesen, J. C. a H., Havermans, R. C., Nederkoorn, C., \& Jansen, A. (2012). Impulsivity in the supermarket. Responses to calorie taxes and subsidies in healthy weight undergraduates. Appetite, 58(1), 6-10.

Gordis, E. B., Granger, D. a, Susman, E. J., \& Trickett, P. K. (2008). Salivary alpha amylasecortisol asymmetry in maltreated youth. Hormones and Behavior, 53(1), 96-103.

Hellhammer, J., \& Schubert, M. (2012). The physiological response to Trier Social Stress Test relates to subjective measures of stress during but not before or after the test.

Psychoneuroendocrinology, 37(1), 119-124.

Hellriegel, E. T., \& D’Mello, a P. (1997). The effect of acute, chronic and chronic intermittent stress on the central noradrenergic system.

Pharmacology, Biochemistry, and Behavior, 57(12), 207-14.

Hoodac, A., Pulversa, K., Spadyb, T. J., Kliebensteina, A., \& Bachanda, J. (2015). Anxiety mediates the effect of acute stress on working memory performance when cortisol levels are high - a moderated mediation analysis. Anxiety, Stress \& Coping: An International Journal, 28(15), 545562.

Jezova, D., Ochedalski, T., Glickman, M., Kiss, a, \& Aguilera, G. (1999). Central corticotropinreleasing hormone receptors modulate hypothalamic-pituitary-adrenocortical and sympathoadrenal activity during stress. Neuroscience, 94(3), 797-802. 
Kang, D. H., \& Fox, C. (2000). Neuroendocrine and leukocyte responses and pulmonary function to acute stressors. Annals of Behavioral Medicine : A Publication of the Society of Behavioral Medicine, 22(4), 276-85.

Keller, K. L. (2012). Strategic brand management: Building, measuring, and managing brand equity (4th ed.). New Jersey: Prentice Hall.

Khalfa, S., Bella, S. D., Roy, M., Peretz, I., \& Lupien, S. (2003). Effects of relaxing music on salivary cortisol level after psychological stress. Annals of the New York Academy of Science, 021, $67-69$.

King, J. a, \& Edwards, E. (1999). Early stress and genetic influences on hypothalamic-pituitaryadrenal axis functioning in adulthood. Hormones and Behavior, 36(2), 79-85.

Kirschbaum, C., \& Hellhammer, D. H. (2000). Salivary Cortisol. In encyclopedia of stress (Vol. 3, pp. 379-383).

Kirschbaum, C., Kudielka, B. M., Gaab, J., Schommer, N. C., \& Hellhammer, D. H. (1999). Impact of gender, menstrual cycle phase, and oral contraceptives on the activity of the hypothalamuspituitary-adrenal axis. Psychosomatic Medicine, 61(2), 154-62.

Kirschbaum, C., Pirke, K., \& Hellhammer, D. (1993). The "Trier Social Stress Test"-a tool for investigating psychobiological stress responses in a laboratory setting. Neuropsychobiology, 2, 76-81.

Knight, W., \& Rickard, N. (2001). Relaxing music prevents stress-induced increases in subjective anxiety, systolic blood pressure, and heart rate in healthy males and females. Journal of Music Therapy, 38(4), 254-272.

Kreibig, S. D. (2010). Autonomic nervous system activity in emotion: a review. Biological Psychology, 84(3), 394-421.

Krieger, D. T. (1975). Rhythms of ACTH and corticosteroid secretion in health and disease, and their experimental modification. Journal of Steroid Biochemistry, 6(5), 785-791.

Kudielka, B. M., Hellhammer, D. H., \& Kirschbaum, C. (2000). sex differences in human stress response. In encyclopedia of stress (Vol. 3).
Levine, S. (2005). Developmental determinants of sensitivity and resistance to stress.

Psychoneuroendocrinology, 30(10), 939-46.

Lighthall, N. R., Gorlick, M. a, Schoeke, A., Frank, M. J., \& Mather, M. (2012). Stress modulates reinforcement learning in younger and older adults. Psychology and Aging, 28(1), 35-46.

Malhotra, N. K. (2001). Pesquisa de Marketinguma orientação aplicada (5 edição). São Paulo: Atlas.

Margis, R., Picon, P., Cosner, A. F., \& Silveira, R. D. O. (2003). Relação entre estressores, estresse e ansiedade. Revista de Psiquiatria, 25(suplemento 1), 65-74.

Marôco, J. (2011). Análise estatística com utilização do SPSS (5 edição). Lisboa: Sílabo.

Mathur, A., Moschis, G. P., \& Lee, E. (2006). Consumer stress-handling strategies: theory and research findings. Journal of Consumer Behaviour, 5(June), 193-203.

McCraty, R., Atkinson, M., Rein, G., \& Watkins, A. (1996). MUSIC ENHANCES THE EFFECT OF POSITIVE EMOTIONAL STATES ON SALIVARI IgA. Stress Medicine, 12, 167-175.

McEwen, B. (2007). Physiology and neurobiology of stress and adaptation: central role of the brain. Physiological Reviews, 873-904.

McNair, D. M., Lorr, M., \& Droppleman, L. F. (1971). Manual for de profile of mood states (POMS). San Diego: Educational and Industrial Testing Services.

Mehta, R., \& Belk, R. W. (1991). Artifacts, identity, and transition: Favorite possessions of Indians and Indian immigrants to the United States. Journal of Consumer Research, 17(4), 398-411.

Mello, A. F., Juruena, M. F., Maciel, M. R., Cavalcante-Nobrega, L. P., Cividanes, G. C., Fossaluza, V., ... Mari, J. de J. (2015). Factors related to the cortisol awakening response of children working on the streets and siblings, before and after 2 years of a psychosocial intervention. Psychiatry Research, 225(3), 625-630.

Mitchell, S., \& Epstein, L. (1996). Changes in Taste and Satiety in Dietary-Restrained Women Following Stress. Physiology \& Behavior, 60(2), 495-9. 
Mommersteeg, P. M. C., Heijnen, C. J., Kavelaars, A., \& van Doornen, L. J. P. (2006). Immune and endocrine function in burnout syndrome. Psychosomatic Medicine, 68(6), 879-86.

Muehlhan, M., Lueken, U., Wittchen, H.-U., \& Kirschbaum, C. (2011). The scanner as a stressor: evidence from subjective and neuroendocrine stress parameters in the time course of a functional magnetic resonance imaging session. International Journal of Psychophysiology : Official Journal of the International Organization of

Psychophysiology, 79(2), 118-26.

Newman, E., O'Connor, D. B., \& Conner, M. (2007). Daily hassles and eating behaviour: the role of cortisol reactivity status.

Psychoneuroendocrinology, 32(2), 125-32.

O’Guinn, T. C., \& Faber, R. J. (1989). Compulsive buying: A phenomenological exploration. Journal of Consumer Research, 16(2), 147-157.

Ottenweller, J. (2000). Animals models (nonprimate) for human stress. In G. Fink (Ed.), Encyclopedia of Stress (p. 2328). New York: Academic Press.

Pacak, K. (2000). Stressor-specific activation of the hypothalamic-pituitary-adrenocortical axis. Physiological Research, 49, 11-17.

Pacak, K., \& McCarty, R. (2000). Acute stress response: experimental. In George Fink (Ed.), Encyclopedia of Stress (p. 2328). New York: Academic Press.

Paris, J., Franco, C., Sodano, R., Freidenberg, B., Gordis, E., Anderson, D. A., ... Frye, C. A. (2010). Sex differences in salivary cortisol in response to acute stressors among healthy participants, in recreational or pathological gamblers, and in those with posttraumatic stress disorder. Hormones and ..., 57(1), 35-45.

Peters, M. L., Godaert, G. L. R., Ballieux, R. E., Vliet, M. van, Willmensen, J. J., Sweep, F. C. G. J., \& Heijnen, C. J. (1998). Cardiovascular a n d endocrine responses to experimental stress: effects of mental effort and controllability. Psychone, 23(I), 1-17.

Read, G. F., Walker, R. F., Wilson, D. W., \& Griffiths, K. (1990). Steroid Analysis in Saliva for the Assessment of Endocrine Function. Annals of the New York Academy of Sciences, 595(1 Steroid Forma), 260-274.
Ribeiro, S. C., Kennedy, S. E., Smith, Y. R., Stohler, C. S., \& Zubieta, J.-K. (2005). Interface of physical and emotional stress regulation through the endogenous opioid system and mu-opioid receptors. Progress in Neuro-Psychopharmacology \& Biological Psychiatry, 29(8), 1264-80.

Rutters, F., Nieuwenhuizen, A. G., Lemmens, S. G. T., Born, J. M., \& Westerterp-Plantenga, M. S. (2009). Acute stress-related changes in eating in the absence of hunger. Obesity (Silver Spring, Md.), 17(1), 72-7.

Sapolsky, R. M., Romero, L. M., \& Munck, A. U. (2000). How Do Glucocorticoids Influence Stress Responses? Integrating Permissive, Suppressive, Stimulatory, and Preparative Actions. Endocrine Reviews, 21(1), 55-89.

Sayette, M. (1993). An appraisal-disruption model of alcohol's effects on stress responses in social drinkers. Psychological Bulletin, 114(3), 459-476.

Smyth, J. M., Ockenfels, M. C., Gorin, A. A., Catley, D., Porter, L. S., Kirschbaum, C., ... Stone, A. A. (1997). Individual differences in the diurnal cycle of cortisol. Psychoneuroendocrinology, 22(2), 89-105.

Smyth, J., Ockenfels, M. C., Porter, L., Kirschbaum, C., Hellhammer, D. H., \& Stone, a a. (1998). Stressors and mood measured on a momentary basis are associated with salivary cortisol secretion. Psychoneuroendocrinology, 23(4), 353-70.

Spielberger, C., \& Sydeman, S. (1994). State-trait anxiety inventory and state-trait anger expression inventory. In M. E. Maruish (Ed.), The use of psychological testing for treatment planning and outcome assessment (p. 637). Hillsdale: Lawrence Erlbaum Associates.

Steptoe, A., Cropley, M., Griffith, J., \& Kirschbaum, C. (2000). Job Strain and Anger Expression Predict Early Morning Elevations in salivary cortisol. Psychosomatic ..., 62, 286-292.

Sterling, P., \& Eyer, J. (1988). Alostasis: a new paradigm to explain arousal pathology. In Handbook of Life Stress, Cognition and Health (pp. 629-649). New York: John Wiley Sons Inc.

Steyer, R., Schwenkmezger, P., Notz, P., Eid, M., 1994. Test- theoretische Analysen des Mehrdimensionalen Befindlich- keitsfragebogens (MDBF). Diagnostica 40, 320-328. 
Strahler, J., Mueller, A., Rosenloecher, F., Kirschbaum, C., \& Rohleder, N. (2010). Salivary alpha-amylase stress reactivity across different age groups. Psychophysiology, 47(3), 587-95.

\section{Stroop, J. R. (1935). STUDIES OF INTERFERENCE IN SERIAL VERBAL REACTIONS. Journal of Experimental Psychology, 18, 643-662.}

Suárez, M., Paglini, P., \& Fernández, R. (1999). Influence of anterodorsal thalamic nuclei on the hypophyseal-adrenal axis and cardiac beta receptors in rats submitted to variable chronic stress. ... : Organo de La ..., 49(2), 71-78.

Taylor, M. J. (2000). Hypothermia. In G. Fink (Ed.), Encyclopedia of Stress (p. 2328). New York: Academic Press.

Thoits, P. a. (1995). Stress, coping, and social support processes: where are we? What next? Journal of Health and Social Behavior, Spec No, 53-79.

Tomiyama, a J., Dallman, M. F., \& Epel, E. S. (2011). Comfort food is comforting to those most stressed: evidence of the chronic stress response network in high stress women.

Psychoneuroendocrinology, 36(10), 1513-9.

Torres, S., Turner, A., \& Nowson, C. (2010). Does stress induce salts intake? British Journal of Nutrition, 103, 1562-1568.

Uchino, B. N., Holt-Lunstad, J., Uno, D., Betancourt, R., \& Garvey, T. S. (1999). Social support and age-related differences in cardiovascular function: an examination of potential mediators. Annals of Behavioral Medicine: A Publication of the Society of Behavioral Medicine, 21(2), 135-42.

Viana, M. F., Almeida, P. L. de, \& Santos, R. C. (2001). Adaptação portuguesa da versão reduzida do Perfil de Estados de Humor - POMS. Análise Psicológica, 1, 77-92.

Von Dawans, B., Kirschbaum, C., \& Heinrichs, M. (2011). The Trier Social Stress Test for Groups (TSST-G): A new research tool for controlled simultaneous social stress exposure in a group format. Psychoneuroendocrinology, 36(4), 514-22.

Weinstock, M. (2007). Gender differences in the effects of prenatal stress on brain development and behaviour. Neurochemical Research, 32(10), 17301740 .

Williams, A. D., \& Grisham, J. R. (2011). Impulsivity, Emotion Regulation, and Mindful Attentional Focus in Compulsive Buying. Cognitive Therapy and Research, 36(5), 451-457.

Wolf, O. T. (2009). Stress and memory in humans: twelve years of progress? Brain Research, 1293, $142-54$.

Zelena, D., Haller, J., Halász, J., \& Makara, G. B. (1999). Social stress of variable intensity: physiological and behavioral consequences. Brain Research Bulletin, 48(3), 297-302.

Zilioli, S., Imami, L., \& Slatcher, R. B. (2015). Life satisfaction moderates the impact of socioeconomic status on diurnal cortisol slope.

Psychoneuroendocrinology, 60, 91-95. 